\title{
Index nominum ad Vol. 160
}

Albeaux-Гernet, M., 392 Althoff, J., 381 Antunes, M. L., 252 Apostolakis, M., 195 (B) Baechler, C, 1, 34 Barbel, L., 300 Bellot, L., 392 (B) Benson, R. C, 195 (B) Bernoth, E., 66 Bertrand, P., 17 Bloch, P., 11 Bonhote, jun., G., 34 Bossart, H., 218 Cahen, C. H., 105 Canato, C, 252 Canet, L., 392 (B) Carpén, E. 0., 315 Cernoch, A., 293 Chappaz, G., 17

Cloeren, S., 269, 281 Cupryn, J., 183 De Jorge, F. B., 252 Delascio, D., 252 Deribreux, J., 392

(B) Geering, P., 166 Gelinet, M., 392 (B) Gétaz, D., 218 Goetschel, E., 94, 344 Goldberg, R., 255 Haefeli, H., 281 Imholz, G., 190 Jäämeri, K. E. U., 315 Kallio, H., 321 Keller, M., 129, 233, 269 Koivuniemi, A. P., 315 Krauer, F., 56 Krauer-Mayer, B., 61 Langen, D., 391 (B) 392

Index nominum ad Vol. 160

Lóránd, S., 300 Ludwig, K. S., 269 Mall-Haefeli, M., 269, 281 Mayer, A., 157, 355 Mohr, U., 381 Morin, P., 391 (B) Orengo, F., 221 Pataki, G., 129 Piukovich, I., 247 Przybora, L. A., 69 Ratzkowski, E., 87 Revaz, C, 333 Rippmann, E. T., 117, 349 Robinson, E., 87 Romani, J.-D., 392 (B) Rozin, S., 360 (B)

S. Sadovsky, E., 49 Saling, E., 133 Schild, W., 39 Schmidt-Mattiesen, H., 197 Schönfelder, K., 361 Scultéty, S., 247 Staemmler, H.-J., 390 (B) Stokvis, B., 391 (B) Thiery, M., 237 Vago, O., 377 Voigt, D., 195 (B) Watteville, H. de, 1 Widholm, O., 321 Willighagen, R. G. J., 237 Wrba, H., 381 Zuckerman, H., 49 